\title{
Atrial Fibrillation/ flutter Hospitalizations among US Medicaid Recipients with and without Systemic Lupus Erythematosus
}

\author{
Sarah K. Chen, Medha Barbhaiya, Daniel H. Solomon, Hongshu Guan, Kazuki Yoshida, \\ Candace H. Feldman, Brendan M. Everett, and Karen H. Costenbader
}

\begin{abstract}
Objective. Systemic lupus erythematosus (SLE) is a multisystem chronic inflammatory autoimmune disease with high prevalence of several risk factors for atrial fibrillation/flutter (AF). However, the incidence and risk of AF in SLE have not been well quantified.

Methods. We used the United States Medicaid Analytic eXtract from 2007 to 2010 to identify beneficiaries aged 18-65 years, with prevalent SLE, each matched by age and sex to 4 non-SLE general Medicaid recipients. We estimated the incidence rates (IR) per 1000 person-years (PY) for AF hospitalizations and used multivariable Cox regression to estimate the HR for AF hospitalization.

Results. We identified 46,876 US Medicaid recipients with SLE, and 187,504 age- and sex-matched non-SLE controls (93\% female; mean age $41.5 \pm 12.2$ yrs). Known AF risk factors such as hypertension (HTN), cardiovascular disease (CVD), and kidney disease were more prevalent in patients with SLE. During a mean followup of $1.9 \pm 1.1$ years for SLE, and $1.8 \pm 1.1$ years for controls, the IR per 1000 PY for AF was 1.4 (95\% CI 1.1-1.6) among patients with SLE and 0.7 (95\% CI 0.6-0.8) among non-SLE controls. In age- and sex-matched and race-adjusted Cox models, the HR for AF was 1.79 (95\% CI 1.43-2.24); after adjustment for baseline HTN and CVD, the adjusted HR was reduced to 1.17 (95\% CI 0.92-1.48).

Conclusion. SLE was associated with a doubled rate of hospitalization for AF compared to age- and sex-matched general Medicaid patients. In a race-adjusted model, the risk was $80 \%$ higher. However, the AF risk factors HTN and CVD were more prevalent among patients with SLE and accounted for the excess risk. (First Release July 1 2020; J Rheumatol 2020;47:1359-65; doi:10.3899/jrheum.190502)
\end{abstract}

Key Indexing Terms:

SYSTEMIC LUPUS ERYTHEMATOSUS

ATRIAL FIBRILLATION

\section{CARDIOVASCULAR DISEASE MEDICAID}

Atrial fibrillation and flutter (AF), common cardiac arrhythmias, are associated with increased morbidity and mortality ${ }^{1,2}$. Systemic lupus erythematosus (SLE) is a multisystem autoimmune disease characterized by autoantibody production and systemic inflammation, as well as a high burden of cardiovascular disease (CVD) $)^{3,4}$. AF is also known to increase the risk of stroke $e^{5,6}$, which is elevated among patients with $\mathrm{SLE}^{7,8,9,10}$. Studies of CVD in SLE patient populations have focused mainly on the outcomes of ischemic heart disease and strokes, with little evidence regarding the risk of AF among SLE compared to the general population $^{11,12}$.

Risk factors for AF, which include hypertension (HTN), obesity, CVD, heart failure, valvular heart disease, and
From the Division of Rheumatology, Immunology and Allergy, the Division of Pharmacoepidemiology and Pharmacoeconomics, and the Divisions of Cardiovascular and Preventive Medicine, Department of Medicine, Brigham and Women's Hospital, Boston, Massachusetts; Division of Rheumatology, Department of Medicine, Hospital for Special Surgery, New York, New York, USA.

This work was funded by the US National Institutes of Health (NIH) RO1 AR057327, K24 AR066109 (Dr. K.H. Costenbader), NIH R56 HL134810 (Dr. B.M. Everett), and NIH-P30-AR072577 (VERITY). Dr. S.K. Chen received research support from NIH T32 AR753033.

S.K. Chen, MD, Division of Rheumatology, Immunology and Allergy, Department of Medicine, Brigham and Women's Hospital; M. Barbhaiya, $M D, M P H$, Division of Rheumatology, Department of Medicine, Hospital for Special Surgery; D.H. Solomon, MD, MPH, Division of Rheumatology, Immunology and Allergy, Department of Medicine, and Division of Pharmacoepidemiology and Pharmacoeconomics, Department of Medicine, Brigham and Women's Hospital; H. Guan, PhD, Division

\begin{abstract}
of Rheumatology, Immunology and Allergy, Department of Medicine, Brigham and Women's Hospital; K. Yoshida, MD, MPH, ScD, Division of Rheumatology, Immunology and Allergy, Department of Medicine, Brigham and Women's Hospital; C.H. Feldman, MD, MPH, ScD, Division of Rheumatology, Immunology and Allergy, Department of Medicine, Brigham and Women's Hospital; B.M. Everett, MD, MPH, Divisions of Cardiovascular and Preventive Medicine, Department of Medicine, Brigham and Women's Hospital; K.H. Costenbader, MD, MPH, Division of Rheumatology, Immunology and Allergy, Department of Medicine, Brigham and Women's Hospital. B.M. Everett and K.H. Costenbader are co-senior authors.

Address correspondence to Dr. S.K. Chen, Division of Rheumatology, Immunology and Allergy, Brigham and Women's Hospital, 60 Fenwood Road, Boston, Massachusetts 02115, USA. E-mail: schen30@bwh.harvard.edu
\end{abstract}

Accepted for publication October 11, 2019.

Personal non-commercial use only. The Journal of Rheumatology Copyright $\subset$ 2020. All rights reserved. 
chronic kidney disease (CKD), are all more prevalent among patients with SLE than in the general population $^{13,14,15}$. Systemic inflammation itself has been implicated in the pathogenesis of $\mathrm{AF}^{16,17,18}$. In other chronic inflammatory diseases, such as inflammatory bowel disease and rheumatoid arthritis, studies have shown an increased risk of AF compared to age- and sex-matched controls, although with conflicting results after adjustment for known AF risk factors ${ }^{19,20,21,22}$.

The objective of our study was to investigate rates, risks, and risk factors for AF among US Medicaid patients with SLE and compare them to those of age- and sex-matched general Medicaid patients. Because patients with SLE have chronically elevated systemic inflammation, we hypothesized that the rates and risks of AF would be elevated among them compared to the general population, related both to known AF risk factors as well as to SLE itself.

\section{MATERIALS AND METHODS}

Data source and cohort definitions. We used the Medicaid Analytic eXtract (MAX), a database that includes billing claims, demographic information, and medication dispensing data for patients enrolled in Medicaid, the US health insurance for low-income individuals. We identified adults aged 18-65 years residing in the 29 most populated states and who were enrolled in Medicaid between January 1, 2007, and December 31, 2010. We did not obtain claims data for patients over age 65 , as they may be dually eligible for both Medicaid and Medicare, and thus they were not included in this study.

Prevalent SLE was defined as having $\geq 3$ International Classification of Diseases, 9th revision (ICD-9) codes for SLE (710.0) from hospital discharge diagnoses or physician visit claims, each $\geq 30$ days apart, as in our prior studies ${ }^{23}$. A prior validation study using Canadian administrative claims showed sensitivity of $98.2 \%$ and specificity of $72.5 \%$ using $\geq 2$ SLE billing diagnoses at least 2 months apart ${ }^{24}$; our algorithm required at least 3 SLE billing diagnoses in an attempt to increase our specificity. We required a 6-month period of continuous enrollment for collection of baseline covariable data prior to the index date, which was defined as the date that our definition of SLE was met. If the date of the third ICD-9 code for SLE occurred before the 6 months of continuous enrollment criteria could be met, the next SLE-related claim allowing for a 6-month baseline period thereafter was defined as the index date.

We matched each patient with SLE by age at index date (within 1 month) and sex to 4 general Medicaid population patients who had ICD-9 codes for any non-SLE diagnoses from hospital discharge diagnoses or physician visit claims on the same index date as the matched patient with $\mathrm{SLE}^{25}$. We required a 6-month baseline period of continuous enrollment prior to the assigned index date, and patients with any SLE ICD-9 codes during the baseline period were excluded from the general Medicaid cohort. For both SLE and general Medicaid cohorts, the baseline period was defined as the 6 months before and including the index date. For both cohorts, we excluded patients with AF during the baseline period.

Baseline covariable assessment. Patient characteristics for all cohorts were collected during the baseline period: age, sex, self-reported race/ethnicity, US region of residence, zip code-level median household income from 2010 US Census data in quartiles as a proxy for socioeconomic status. Using ICD-9, Diagnosis Related Group code, and/or Current Procedural Terminology codes, we collected covariables in the baseline period, including the number of outpatient physician visits, smoking, obesity, HTN, diabetes, hyperlipidemia, thyroid disease, CKD, chronic obstructive pulmonary disease (COPD), heart failure, valvular disease, cardiac surgery, and pericarditis/myocarditis using $\geq 1$ ICD-9 $\operatorname{code}^{1,26,27,28,29,30}$. In the SLE cohort, patients with lupus nephritis (LN) were defined as having $\geq 2$ ICD-9 hospital discharge diagnoses or physician billing claims for nephritis, proteinuria, and/or renal failure, occurring $\geq 30$ days apart, on or after the SLE criteria were met $^{31,32}$. CVD at baseline was defined as the presence of any of the following covariables during the baseline period: acute myocardial infarction, old myocardial infarction, angina, coronary atherosclerosis, percutaneous coronary intervention, coronary artery bypass graft, cerebrovascular accident, peripheral vascular disease, carotid artery stenosis, and heart failure ${ }^{33-40}$.

We calculated a Charlson Comorbidity Index for all patients and SLE-specific risk adjustment index for patients with $\mathrm{SLE}^{41,42}$. We identified filled prescriptions using National Drug Codes and summed the number of unique medications filled per subject during the baseline period. We assessed baseline prescriptions filled for anticoagulants, beta blockers, statins, and angiotensin-converting enzyme inhibitor/angiotensin receptor blockers. We assessed prescriptions for glucocorticoid (GC) use (prednisone, methylprednisolone, dexamethasone, hydrocortisone, prednisolone, and cortisone defined as prednisone equivalents), hydroxychloroquine, and immunosuppressive drugs (mycophenolate mofetil, mycophenolic acid, cyclophosphamide, azathioprine, cyclosporine, methotrexate, leflunomide, rituximab, and tacrolimus).

Outcomes. The primary outcome was incident AF hospitalization defined by ICD-9 codes 427.31 (atrial fibrillation) and 427.32 (atrial flutter) for either primary or secondary hospital discharge diagnosis codes ${ }^{43}$. Patients were followed from index date until first AF hospitalization, death, disenrollment, or end of followup.

Statistical methods. We calculated unadjusted AF incidence rates (IR) per 1000 person-years (PY) with $95 \%$ CI. We used proportional hazards models accounting for competing risk of death to estimate the subdistribution HR for incident AF hospitalization in SLE, compared to the general Medicaid cohort in sequential models, examining potential confounders of the association between SLE and $\mathrm{AF}^{44}$. We first ran an age- and sex-matched model adjusted for race/ethnicity in model 1 . To build parsimonious models given the low number of events, we examined potential confounders individually to identify variables that changed the point estimate by $>10 \%$. In model 2 , we additionally adjusted for the 2 variables that had the largest effect on the point estimate (CVD and HTN). Adjustment for other covariates that changed the point estimate by $>10 \%$ did not further influence risk estimates. We also stratified patients with SLE by baseline GC use (yes/no) and ran our final Cox model in each group.

We performed all analyses using SAS version 9.4 (SAS Institute Inc.) using data obtained from the Centers for Medicare and Medicaid Services through approved Data Use Agreements, and data are presented according to federal reporting guidelines. The Partners' Institutional Review Board approved all aspects of this study (IRB\# 2013P002602).

\section{RESULTS}

The SLE cohort included 46,876 patients and the general Medicaid cohort included 187,504 patients (Table 1). The mean age for patients in both cohorts was 41.5 ( \pm SD 12.2) years and $93 \%$ were female. The mean followup durations were $1.9( \pm 1.1)$ years for SLE and $1.8( \pm 1.1)$ years for the general Medicaid cohorts. A higher proportion of SLE patients were African American by self-report compared to the general Medicaid population (42\% compared to $22 \%$ ). Patients with SLE had higher prevalence of baseline HTN, hyperlipidemia, diabetes mellitus, smoking, valvular disease, CVD, thyroid disease, and use of anticoagulants, beta blockers, and statins compared to the general Medicaid cohort. Among the SLE cohort, $41 \%$ used GC during the

Personal non-commercial use only. The Journal of Rheumatology Copyright (c) 2020. All rights reserved. 
Table 1. Baseline* characteristics for SLE, age- and sex-matched general Medicaid cohorts, 2007-2010.

\begin{tabular}{|c|c|c|}
\hline Characteristics & SLE & General Medicaid \\
\hline Matched cohort size, $\mathrm{N}$ & 46,876 & 187,504 \\
\hline Female & 93 & 93 \\
\hline Age, yrs, mean (SD) & $41.5(12.2)$ & $41.5(12.2)$ \\
\hline \multicolumn{3}{|l|}{ US region of residence } \\
\hline West & 19 & 27 \\
\hline Northeast & 20 & 19 \\
\hline South & 41 & 34 \\
\hline Midwest & 20 & 20 \\
\hline \multicolumn{3}{|l|}{ Race/Ethnicity } \\
\hline White & 35 & 46 \\
\hline African American & 42 & 22 \\
\hline Hispanic & 16 & 25 \\
\hline Asian & 3 & 3 \\
\hline American Indian/Alaskan Native & 1 & 1 \\
\hline Lupus nephritis/CKD & 19 & 2 \\
\hline Hypertension & 37 & 15 \\
\hline Hyperlipidemia & 12 & 7 \\
\hline Obesity & 5 & 4 \\
\hline Smoking & 7 & 4 \\
\hline $\mathrm{CVD}^{\dagger}$ & 16 & 6 \\
\hline Heart failure & 7 & 2 \\
\hline Diabetes mellitus & 14 & 10 \\
\hline Valvular disease & 6 & 1 \\
\hline Pericarditis/myocarditis & 1 & $<1$ \\
\hline Cardiac surgery & 6 & 2 \\
\hline Thyroid disease & 8 & 3 \\
\hline COPD & 12 & 5 \\
\hline No. outpatient visits, mean (SD) & $4.7(4.8)$ & $1.9(3.1)$ \\
\hline No. medications, mean (SD) & $10.7(10.3)$ & $3.9(6.0)$ \\
\hline Anticoagulant use & 6 & $<1$ \\
\hline Beta blocker use & 12 & 5 \\
\hline Statin use & 10 & 6 \\
\hline ACE inhibitor/ARB use & 21 & 8 \\
\hline Hydroxychloroquine use & 36 & $<1$ \\
\hline Immunosuppressants & 19 & $<1$ \\
\hline Glucocorticoid use $\geq 10 \mathrm{mg} /$ day ever & 41 & 6 \\
\hline Charlson Comorbidity Index, mean (SD) & $2.0(1.5)$ & $0.5(1.3)$ \\
\hline
\end{tabular}

Values are \% unless otherwise specified. * Baseline is the 6-month period prior to and including the index date. CVD: baseline presence of any CVD by ICD-9 codes for acute MI, old MI, angina, coronary atherosclerosis, percutaneous coronary intervention, coronary artery bypass graft, cerebrovascular accident, peripheral vascular disease, carotid artery stenosis, heart failure. ${ }^{\ddagger}$ Immunosuppressants: mycophenolate mofetil, mycophenolic acid, cyclophosphamide, azathioprine, cyclosporine, methotrexate, leflunomide, rituximab, and tacrolimus. SLE: systemic lupus erythematosus; CKD: chronic kidney disease; CVD: cardiovascular disease; ACE: angiotensin-converting enzyme; ARB: angiotensin receptor blocker; COPD: chronic obstructive pulmonary disease; ICD-9: International Classification of Diseases, 9th revision; MI: myocardial infarction.

baseline period compared to $6 \%$ of the general Medicaid cohort.

During the followup period, there were 121 AF hospitalization events in the SLE cohort (IR 1.4 per 1000 PY, 95\% CI 1.1-1.6) and 241 AF hospitalization events in the general Medicaid cohort (IR 0.7 per 1000 PY, 95\% CI 0.6-0.8; Table 2). The IR of AF was higher in males and increased with age in stratified age groups for both SLE and non-SLE patients and remained higher in the SLE cohort across all age categories compared to general Medicaid. The IR of AF for both cohorts was higher among patients with HTN, valvular disease, LN/renal disease, and CVD.

In race/ethnicity-adjusted Cox regression model, the risk of incident $\mathrm{AF}$ hospitalization among patients with SLE was 1.79 -fold higher (95\% CI 1.43-2.24) than the age- and sex-matched general Medicaid population (Figure 1). We identified baseline CVD, HTN, LN/CKD, valvular disease, and Charlson Comorbidity Index as variables that changed the point estimate by $>10 \%$ when each was separately added to the race/ethnicity adjusted model. The strongest attenuation in HR was seen when additionally adjusted for CVD and HTN. The risk estimate was attenuated and became

Personal non-commercial use only. The Journal of Rheumatology Copyright @ 2020 . All rights reserved. 
Table 2. Incidence rates for atrial fibrillation/flutter within the SLE and general Medicaid cohorts from 2007 to 2010 by baseline covariates.

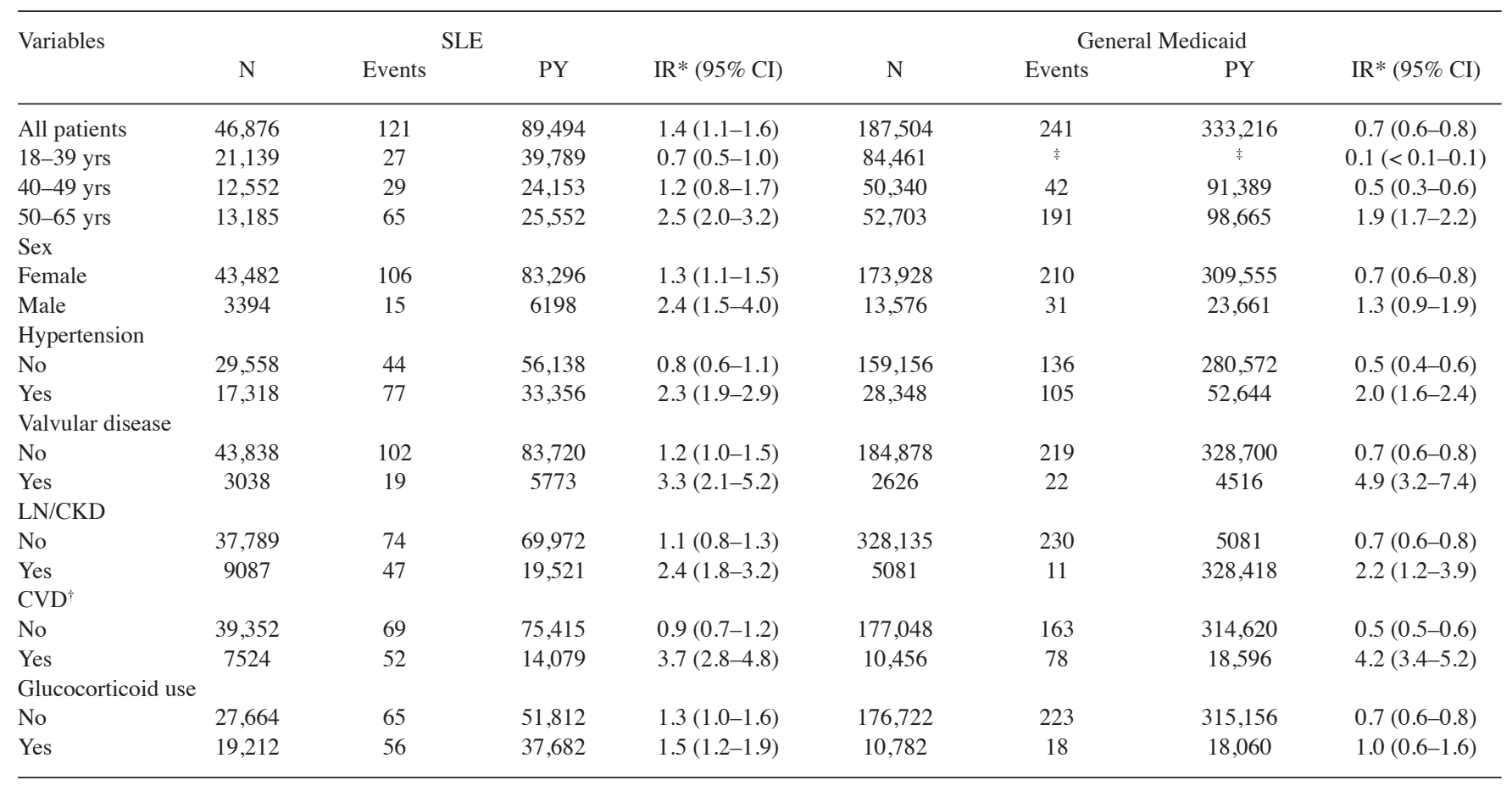

* IR: per 1000 PY. ${ }^{\dagger}$ CVD: baseline presence of any CVD by ICD-9 codes for angina, MI, old MI, percutaneous coronary intervention, atherosclerosis, cerebrovascular accident, coronary artery bypass graft, peripheral vascular disease, carotid stenosis, heart failure. ${ }^{\ddagger}$ Cell sizes under 11 are suppressed according to Federal Reporting Requirements. SLE: systemic lupus erythematosus; PY: person-years; IR: incidence rate; CVD: cardiovascular disease; CKD: chronic kidney disease; ICD-9: International Classification of Diseases, 9th revision; MI: myocardial infarction; LN: lupus nephritis.

non-significant in a combined model adjusting for race/ ethnicity, CVD, and HTN (HR 1.17, 95\% CI 0.92-1.48). No further adjustment affected risk estimates. Additionally, when we stratified SLE patients by baseline GC use, there was no significant increased risk of AF in SLE patients with and without baseline GC use in our adjusted models.

\section{DISCUSSION}

In this large cohort study of US Medicaid patients, the IR of hospitalization for AF among patients with SLE was about double that of Medicaid patients matched for age and sex who did not have SLE. This increased rate of AF for patients with SLE was observed across all age groups. The relative risk of AF among patients with SLE was nearly $80 \%$ higher than that of age- and sex-matched general Medicaid population controls when adjusted for race/ethnicity. Most of the excess risk of AF among patients with SLE was accounted for by high prevalence of HTN and CVD, and to a lesser extent by renal disease, valvular disease, and Charlson Comorbidity Index. Thus, SLE is associated with increased prevalence of important risk factors for AF, including HTN and CVD, which appear to be responsible for the increased incidence of AF hospitalizations observed.

These results have clinical and epidemiological significance for several reasons. This Medicaid cohort is the largest to have been assessed for incident AF across a broad age range of patients with SLE, to our knowledge. In a retrospective study of 235 patients with SLE at a single academic center, investigators reported a prevalence of $9 \%$ for atrial fibrillation and $3 \%$ for atrial flutter ${ }^{45}$. A phenome-wide association study of 1097 patients with SLE demonstrated an association between SLE disease status and AF, and increased incidence of AF in male patients, a finding also seen in our SLE and general Medicaid patients ${ }^{11}$. However, the phenome-wide association study was unable to account for potential mediating factors such as HTN and valvular heart disease.

In a recent study by Lim, et al using the Korean National Health Insurance Service National Sample Cohort database from 2008 to 2014, the IR of AF among 21,143 patients with SLE was 3.69 compared to 0.94 per 1000 PY in ageand sex-matched non-SLE controls ${ }^{12}$. The Korean study included both inpatient and outpatient AF, which likely explains the much higher rates of AF in both the SLE and non-SLE controls compared to our study. After multivariable adjustment, the HR for AF remained 2.84-fold higher than that of controls (95\% CI 2.50-3.23) in the study by Lim, et al. While their multivariable models adjusted for age, sex, income, and many relevant comorbidities (diabetes, HTN, dyslipidemia, COPD, endstage renal disease, myocardial infarction, stroke, and heart failure), the definition of CVD used in our multivariable adjustment included more CV variables.

Personal non-commercial use only. The Journal of Rheumatology Copyright @ 2020 . All rights reserved. 
MODEL 2

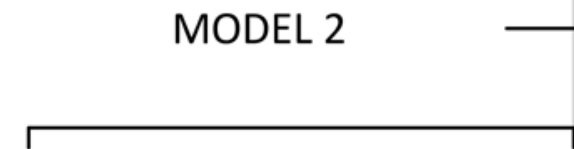

0.5

Figure 1. Subdistribution HR (HRsd)* and 95\% CI for incident hospitalization for atrial fibrillation/flutter among patients with SLE compared to an age- and sex-matched general Medicaid population. Model 1: matched on age and sex + adjusted for race/ethnicity. Model 2: Model $1+$ hypertension and CVD. *HRsd: HR for first atrial fibrillation/flutter hospitalization event, competing risk analysis taking risk of death into account. SLE: systemic lupus erythematosus; CVD: cardiovascular disease.

Our hypothesis that the risk of hospitalizations for AF would be higher among patients with SLE was based on several studies that have implicated the role of inflammation in the pathogenesis of AF. Cardiac biopsies from patients with $\mathrm{AF}$ have demonstrated inflammatory infiltrates on histology, and have also been observed in animal models with $\mathrm{AF}^{46,47}$. Key markers of inflammation, including interleukin 6, C-reactive protein, and tumor necrosis factor- $\alpha$, have also been found to be associated with $\mathrm{AF}^{16,17,48,49}$, and are also known to be dysregulated in $\mathrm{SLE}^{50}$. Additionally, in a study of AF risk among inflammatory bowel disease patients, elevated AF risk was significantly higher during active flares and persistent activity, while there was decreased AF risk observed during periods of remission, further supporting the notion that active inflammation may be involved in the pathogenesis of $\mathrm{AF}^{20}$.

We found that the elevated IR observed in SLE was related to the increased prevalence of HTN, valvular disease, LN/ $\mathrm{CKD}$, and CVD compared to the overall general Medicaid population. HTN is one of the most important risk factors for AF and adjusting for HTN and baseline CVD accounted for a substantial portion of the elevated risk for incident $\mathrm{AF}$ hospitalization. Including HTN and CVD in the model attenuated the elevated risk for incident AF hospitalization. Our finding of attenuated risk after accounting for traditional $\mathrm{AF}$ risk factors do not discount the possibility that SLE-related inflammation plays an important role in increasing the risk of AF. Lupus nephritis, valvular disease, and CVD all represent states of high disease activity and inflammation in SLE, and thus are likely intermediates on the pathway to higher risk of AF.

GC may also affect the risk of AF, but we observed no significant increase in AF risk among patients with SLE in analyses stratified by GC use during the 6-month baseline period. While it is possible that GC dose may affect the risk of AF, GC are frequently used in SLE management (41\% of our cohort) but rarely used in non-SLE controls (6\%). Thus, we did not include GC dose in our main analysis comparing the risk of AF in SLE to non-SLE controls, because this would additionally adjust for SLE cohort status given the frequent use of GC in patients with SLE compared to the general Medicaid controls. Additionally, how the use and dose of GC may mediate the association between SLE and $\mathrm{AF}$ is of interest for future studies.

Apart from inflammation and GC use, it is possible that conditions such as valvular disease and renal disease may promote higher AF risk by structural and metabolic mechanisms, respectively. Further, it is unknown how the presence of antiphospholipid antibodies or the use of nonsteroidal antiinflammatory drugs that are not reliably collected in claims studies, contribute to the risk of AF.

Our study has several key strengths and some limitations. We used ICD-9 codes to identify AF with reported sensitivity of $95 \%$, specificity of $99 \%{ }^{43}$, and identified AF hospitalizations with primary or secondary codes without the use of outpatient claims. This likely decreased the sensitivity of identifying all incident $\mathrm{AF}$, particularly because patients may present with symptoms of AF and occasionally are managed solely in the outpatient setting without requiring hospitalization. We did not include outpatient AF because algorithms for its identification have not been validated and prevalent AF diagnoses may be repeatedly observed in claims data. Thus our outcome was restricted to AF that led to hospitalization and decreased the risk of ascertainment bias because age- and sex-matched non-SLE patients may have less contact with the healthcare system, as noted by the lower frequency of outpatient visits among those without SLE in the baseline period. However, adjustment for the number of outpatient visits in our models did not significantly change our effect estimates.

Our SLE cohort was a prevalent cohort in which we excluded those with baseline AF to assess risk for incident AF. Given the short duration of followup and use of hospitalization codes only, these elevated IR for AF among patients with SLE are likely underestimates. We defined baseline covariates by at least 1 ICD-9 code, which may not have accurately identified these covariates. For example, covariates without billing codes during the 6-month baseline

Personal non-commercial use only. The Journal of Rheumatology Copyright $(\subset) 2020$. All rights reserved. 
period would not be identified, while alternatively, we may have recorded some covariates using 1 ICD-9 code that may have been used for billing while ruling out a diagnosis. Owing to the characteristics of administrative data, we were unable to assess SLE disease duration, disease activity, or active inflammation. This is of importance because periods of flare activity and inflammation are likely to be involved in driving the risk of AF. We used a validated algorithm for defining $\mathrm{LN}^{32}$. However, the algorithm potentially does not identify all patients with $\mathrm{LN}$ because of the number of codes required to be classified. Further, most Medicaid patients who develop endstage renal disease from SLE or other causes change insurance coverage to Medicare and thus these claims could not be recorded. Our study was conducted in US Medicaid recipients and we excluded patients over age 65 because we do not have claims data for patients dually eligible for both Medicaid and Medicare. While the Medicaid program represents a significant percentage of the US population with a high burden of chronic diseases including SLE, this may limit the generalizability of our findings for patients with commercial insurance and those of older age.

We found an increased incidence of $\mathrm{AF}$ among patients with SLE enrolled in Medicaid compared to an age- and sex-matched general Medicaid population. The risk of AF for patients with SLE was double of that for patients without SLE. This risk appeared to be due to the associations between SLE and common risk factors for AF, including HTN and CVD. Further work should target modifying the risk factors for AF identified in this study in an effort to decrease the risk of AF among patients with SLE.

\section{ACKNOWLEDGMENT}

We thank Emma Stevens for technical review and Chang Xu for programming review of this manuscript.

\section{REFERENCES}

1. Go AS, Hylek EM, Phillips KA, Chang Y, Henault LE, Selby JV, et al. Prevalence of diagnosed atrial fibrillation in adults: national implications for rhythm management and stroke prevention: the AnTicoagulation and Risk Factors in Atrial Fibrillation (ATRIA) Study. JAMA 2001;285:2370-5.

2. Benjamin EJ, Wolf PA, D'Agostino RB, Silbershatz H, Kannel WB, Levy D. Impact of atrial fibrillation on the risk of death: the Framingham Heart Study. Circulation 1998;98:946-52.

3. Hak EA, Karlson EW, Feskanich D, Stampfer MJ, Costenbader $\mathrm{KH}$. Systemic lupus erythematosus and the risk of cardiovascular disease: results from the nurses' health study. Arthritis Rheum 2009;61:1396-402

4. Bartels CM, Buhr KA, Goldberg JW, Bell CL, Visekruna M, Nekkanti S, et al. Mortality and cardiovascular burden of systemic lupus erythematosus in a US population-based cohort. J Rheumatol 2014;41:680-7.

5. Wolf PA, Abbott RD, Kannel WB. Atrial fibrillation as an independent risk factor for stroke: the Framingham Study. Stroke 1991;22:983-8

6. Risk factors for stroke and efficacy of antithrombotic therapy in atrial fibrillation. Analysis of pooled data from five randomized controlled trials. Arch Intern Med 1994;154:1449-57.
7. Esdaile JM, Abrahamowicz M, Grodzicky T, Li Y, Panaritis C, du Berger R, et al. Traditional Framingham risk factors fail to fully account for accelerated atherosclerosis in systemic lupus erythematosus. Arthritis Rheum 2001;44:2331-7.

8. Chiu CC, Huang CC, Chan WL, Chung CM, Huang PH, Lin SJ, et al. Increased risk of ischemic stroke in patients with systemic lupus erythematosus: a nationwide population-based study. Intern Med 2012;51:17-21.

9. Aviña-Zubieta JA, To F, Vostretsova K, De Vera M, Sayre EC, Esdaile JM. Risk of myocardial infarction and stroke in newly diagnosed systemic lupus erythematosus: a general population-based study. Arthritis Care Res 2017;69:849-56.

10. Barbhaiya M, Feldman CH, Guan H, Chen SK, Fischer MA, Solomon DH, et al. Racial/ethnic variation in stroke rates and risks among patients with systemic lupus erythematosus. Semin Arthritis Rheum 2019;48:840-6.

11. Barnado A, Carroll RJ, Casey C, Wheless L, Denny JC, Crofford LJ. Phenome-wide association studies uncover a novel association of increased atrial fibrillation in male patients with systemic lupus erythematosus. Arthritis Care Res 2018;70:1630-6.

12. Lim SY, Bae EH, Han KD, Jung JH, Choi HS, Kim CS, et al. Systemic lupus erythematosus is a risk factor for atrial fibrillation: a nationwide, population-based study. Clin Exp Rheumatol 2019;37:1019-25.

13. Benjamin EJ, Levy D, Vaziri SM, D'Agostino RB, Belanger AJ, Wolf PA. Independent risk factors for atrial fibrillation in a population-based cohort. The Framingham Heart Study. JAMA 1994;271:840-4.

14. Wang TJ, Parise H, Levy D, D'Agostino RB, Wolf PA, Vasan RS, et al. Obesity and the risk of new-onset atrial fibrillation. JAMA 2004;292:2471.

15. Alonso A, Lopez FL, Matsushita K, Loehr LR, Agarwal SK, Chen $\mathrm{LY}$, et al. Chronic kidney disease is associated with the incidence of atrial fibrillation: the Atherosclerosis Risk in Communities (ARIC) Study. Circulation 2011;123:2946-53.

16. Chung MK, Martin DO, Sprecher D, Wazni O, Kanderian A, Carnes CA, et al. C-reactive protein elevation in patients with atrial arrhythmias: inflammatory mechanisms and persistence of atrial fibrillation. Circulation 2001;104:2886-91.

17. Aviles RJ, Martin DO, Apperson-Hansen C, Houghtaling PL, Rautaharju P, Kronmal RA, et al. Inflammation as a risk factor for atrial fibrillation. Circulation 2003;108:3006-10.

18. Engelmann MD, Svendsen JH. Inflammation in the genesis and perpetuation of atrial fibrillation. Eur Heart J 2005;26:2083-92.

19. Lindhardsen J, Ahlehoff O, Gislason GH, Madsen OR, Olesen $\mathrm{JB}$, Svendsen JH, et al. Risk of atrial fibrillation and stroke in rheumatoid arthritis: Danish nationwide cohort study. BMJ 2012;344:e1257.

20. Kristensen SL, Lindhardsen J, Ahlehoff O, Erichsen R, Lamberts M, Khalid U, et al. Increased risk of atrial fibrillation and stroke during active stages of inflammatory bowel disease: a nationwide study. Europace 2014;16:477-84.

21. Kim SC, Liu J, Solomon DH. The risk of atrial fibrillation in patients with rheumatoid arthritis. Ann Rheum Dis 2014;73: 1091-5.

22. Bacani AK, Crowson CS, Roger VL, Gabriel SE, Matteson EL. Increased incidence of atrial fibrillation in patients with rheumatoid arthritis. Biomed Res Int 2015;2015:809514.

23. Feldman CH, Yazdany J, Guan H, Solomon DH, Costenbader KH. Medication nonadherence is associated with increased subsequent acute care utilization among medicaid beneficiaries with systemic lupus erythematosus. Arthritis Care Res 2015;67:1712-21.

24. Bernatsky S, Linehan T, Hanly JG. The accuracy of administrative data diagnoses of systemic autoimmune rheumatic diseases. J Rheumatol 2011;38:1612-6. 
25. Mayo Clinic. Division of Biomedical Statistics and Informatics, Mayo Clinic Research. Biomedical statistics and informatics software packages. [Internet. Accessed June 12, 2020.] Available from: http://bioinformaticstools.mayo.edu/research/gmatch

26. Wiley LK, Shah A, Xu H, Bush WS. ICD-9 tobacco use codes are effective identifiers of smoking status. J Am Med Inform Assoc 2013;20:652-8.

27. Quan H, Li B, Saunders LD, Parsons GA, Nilsson CI, Alibhai A, et al; IMECCHI Investigators. Assessing validity of ICD-9-CM and ICD-10 administrative data in recording clinical conditions in a unique dually coded database. Health Serv Res 2008;43:1424-41.

28. Quan H, Sundararajan V, Halfon P, Fong A, Burnand B, Luthi JC, et al. Coding algorithms for defining comorbidities in ICD-9-CM and ICD-10 administrative data. Med Care 2005;43:1130-9.

29. Chronic Conditions Data Warehouse. Condition Categories. [Internet. Accessed March 9, 2020.] Available from: www.ccwdata. org/web/guest/condition-categories

30. Winkelmayer WC, Schneeweiss S, Mogun H, Patrick AR, Avorn J, Solomon DH. Identification of individuals with CKD from Medicare claims data: a validation study. Am J Kidney Dis 2005;46:225-32.

31. Feldman CH, Hiraki LT, Liu J, Fischer MA, Solomon DH, Alarcón GS, et al. Epidemiology and sociodemographics of systemic lupus erythematosus and lupus nephritis among US adults with Medicaid coverage, 2000-2004. Arthritis Rheum 2013;65:753-63.

32. Chibnik L, Massarotti E, Costenbader K. Identification and validation of lupus nephritis cases using administrative data. Lupus 2010;19:741-3.

33. Liao KP, Liu J, Lu B, Solomon DH, Kim SC. Association between lipid levels and major adverse cardiovascular events in rheumatoid arthritis compared to non-rheumatoid arthritis patients. Arthritis Rheumatol 2015;67:2004-10.

34. Solomon DH, Schneeweiss S, Glynn RJ, Kiyota Y, Levin R, Mogun $\mathrm{H}$, et al. Relationship between selective cyclooxygenase-2 inhibitors and acute myocardial infarction in older adults. Circulation 2004;109:2068-73.

35. Varas-Lorenzo C, Castellsague J, Stang MR, Tomas L, Aguado J, Perez-Gutthann S. Positive predictive value of ICD-9 codes 410 and 411 in the identification of cases of acute coronary syndromes in the Saskatchewan Hospital automated database. Pharmacoepidemiol Drug Saf 2008;17:842-52.

36. Birman-Deych E, Waterman AD, Yan Y, Nilasena DS, Radford MJ, Gage BF. Accuracy of ICD-9-CM codes for identifying cardiovascular and stroke risk factors. Med Care 2005;43:480-5.

37. Kumamaru H, Judd SE, Curtis JR, Ramachandran R, Hardy NC, Rhodes JD, et al. Validity of claims-based stroke algorithms in contemporary Medicare data: reasons for geographic and racial differences in stroke (REGARDS) study linked with Medicare claims. Circ Cardiovasc Qual Outcomes 2014;7:611-9.

38. Fan J, Arruda-Olson AM, Leibson CL, Smith C, Liu G, Bailey $\mathrm{KR}$, et al. Billing code algorithms to identify cases of peripheral artery disease from administrative data. J Am Med Inform Assoc 2013;20:e349-54.

39. Chen J, Hsieh AF, Dharmarajan K, Masoudi FA, Krumholz HM. National trends in heart failure hospitalization after acute myocardial infarction for Medicare beneficiaries: 1998-2010. Circulation 2013;128:2577-84

40. Idowu RT, Carnahan R, Sathe NA, McPheeters ML. A systematic review of validated methods to capture myopericarditis using administrative or claims data. Vaccine 2013;31 Suppl 1:K34-40.

41. Charlson M, Szatrowski TP, Peterson J, Gold J. Validation of a combined comorbidity index. J Clin Epidemiol 1994;47:1245-51.

42. Ward MM. Development and testing of a systemic lupus-specific risk adjustment index for in-hospital mortality. J Rheumatol 2000;27:1408-13.

43. Glazer NL, Dublin S, Smith NL, French B, Jackson LA, Hrachovec $\mathrm{JB}$, et al. Newly detected atrial fibrillation and compliance with antithrombotic guidelines. Arch Intern Med 2007;167:246-52.

44. Fine JP, Gray RJ. A proportional hazards model for the subdistribution of a competing risk. J Am Stat Assoc 1999; 94:496-509.

45. Myung G, Forbess LJ, Ishimori ML, Chugh S, Wallace D, Weisman $\mathrm{MH}$. Prevalence of resting-ECG abnormalities in systemic lupus erythematosus: a single-center experience. Clin Rheumatol 2017;36:1311-6.

46. Frustaci A, Chimenti C, Bellocci F, Morgante E, Russo MA, Maseri A. Histological substrate of atrial biopsies in patients with lone atrial fibrillation. Circulation 1997;96:1180-4.

47. Nakamura Y, Nakamura K, Fukushima-Kusano K, Ohta K, Matsubara $\mathrm{H}$, Hamuro T, et al. Tissue factor expression in atrial endothelia associated with nonvalvular atrial fibrillation: possible involvement in intracardiac thrombogenesis. Thromb Res 2003;111:137-42.

48. Guo Y, Lip GYH, Apostolakis S. Inflammatory biomarkers and atrial fibrillation: potential role of inflammatory pathways in the pathogenesis of atrial fibrillation-induced thromboembolism. Curr Vasc Pharmacol 2015;13:192-201.

49. Patel P, Dokainish H, Tsai P, Lakkis N. Update on the association of inflammation and atrial fibrillation. J Cardiovasc Electrophysiol 2010;21:1064-70.

50. Aringer M, Smolen JS. Tumour necrosis factor and other proinflammatory cytokines in systemic lupus erythematosus: a rationale for therapeutic intervention. Lupus 2004;13:344-7. 